\title{
Data processing for safety control of bridges in real time
}

\author{
V. Marecos \\ ISEL, Lisbon, Portugal \\ L.O. Santos \\ LNEC, Lisbon, Portugal \\ F. Branco \\ IST, Lisbon, Portugal
}

\begin{abstract}
This paper describes the developed methodologies for data processing of bridge monitoring data in real time, applied to vertical displacement data on Salgueiro Maia Bridge.
\end{abstract}

\section{INTRODUCTION}

The observation of structure's behaviour, especially related to long-term monitoring, has had large improvements with the introduction of new techniques for automatic acquisition of measurements. Automatic acquisition gives the possibility of reading several equipments continuously, simultaneously, without the presence of operators, plus the capability of establishing a remote connection to a centre of studies through phone, radio, GSM or Internet.

For a better use of the automatic acquisition and considering the new developments associated to sensors based on recent technologies, and considering also the upgrading of storage and acquisition equipment, it became necessary the development of new methodologies for data processing.

For the data management and analysis, it is needed to get reliable measurements from each sensor and information on the relations between different sensors and on the evolution of the data with time.

Some of this information is now possible to get directly from the data-loggers with the definition of routines that are introduced on the acquisition program. This way they are done immediately after the readings, and it is possible to repeat some measurements if any anomalous data is detected. These procedures include validation of signals, measurements and tendencies.

On the other hand, to guarantee the observation of the structure in real time, alert and alarm levels have to be established to control, permanently and in situ, the development of selected variables, allowing a more efficient evaluation of safety conditions of the structure.

This paper will describe the developed methodologies for data processing of bridge monitoring data, in real time. These methodologies will be applied to process vertical displacement data on Salgueiro Maia Bridge.

\section{INTEGRATED MONITORING SYSTEM}

The Integrated Monitoring System is divided in three sub systems: Data Acquisition and Processing in real time, Data Transfer and Data Management and Analysis (Marecos et al. 2004).

Data Acquisition and Processing in real time consists in the acquisition of sensor signals, validation of the measurements based on statistical methods and on estimated values for the variable. This sub-system is explained in more detail in section 3.

Data Transfer was created to allow that the data is acquired in the field may be available in the office where it will be analysed by the engineers in real time. The modem not only allows 
remote assessment to the measurements, but also makes it possible to establish connections in the office with the data-loggers to make changes on the programs defined.

Data Management and Analysis is a system that manages all the data collected from the sensors installed on the structure, regularly analyses them and evaluates its time development. The conclusions about structure's safety conditions are published in a periodical report.

\section{DATA ACQUISITION AND PROCESSING}

In data acquisition two different classifications have to be distinguished: data logging and real time data acquisition. In data logging the information is acquired and stored for later used, on the other hand, in real time data acquisition the data is used immediately after being acquired to manage and control the system (Datataker, 2005).

Sensor signals are read and stored with data-loggers which are able to execute measurements without the need for human orders. With the data-logger it is possible to have measurements from all the sensors distributed along the structure simultaneously, allowing the right comparison of the data because they are referred to the same moment in time. Measurement's rate can be specified according to the variability of monitored data. Data-loggers can be linked to each other's building a local network that is commanded by the master data-logger.

After acquiring and validating the data, the data-logger starts the analysis by checking if the value read is under the alert or alarm level and if not, unchains the alert or alarm procedures. Those procedures include the increase of readings frequencies, but also the sending of messages to the technician in charge about anomalous data, allowing him to do more detailed analysis.

\subsection{Data validation}

In order to obtain reliable data for analysis it is necessary to validate the records by verifying if they are within the limits defined and eliminating erroneous ones.

Erroneous data may appear due to several causes, and may be classified according to it's origin in systematic and random errors. Systematic errors are caused by deficiencies in equipment calibration or on the method used originating the replication of the error in all observations. In order to reduce this type of errors all sensors must be calibrated using different methods for the measurement of the same data. Random errors are attributable to various sources such as a breakdown in energy supplies, deficiencies on sensor/data-logger connections or equipment damage. Since this errors occur due to a number of uncontrolled variables they can not be eliminated, but can be minimized using statistical techniques (Aktan et al., 2002).

First stage of data validation consists on verifying if the value read is within measuring range and, if that is not the case, the measure is tagged as impossible and should not be used in future analysis.

The next step corresponds to signal validation that is the detection and elimination of outliers, values that diverge from the majority of monitored data, which can mask structure's response and set up an alarm by mistake.

There are several methods used to detect outliers and the choice of the appropriate method depends on the sample distribution, if the parameters of the distribution are known, the number and type of outliers to be expected (Zhang et al., 2004).

The method used for outlier detection was the modified z-score test, equation (1). The changes from the normal z-score method consisted on the substitution of the mean for the median, and as for the standard deviation it was replaced by three alternatives $\mathrm{MAD}_{n}, \mathrm{~S}_{\mathrm{n}}$ or $\mathrm{Q}_{\mathrm{n}}$.

$$
Z_{i}^{*}=\frac{\left|x_{i}-\widetilde{x}\right|}{M_{n} \text { or } S_{n} \text { or } Q_{n}} \quad\left\{\begin{array}{l}
\operatorname{MAD}_{n}=1.4826 \operatorname{median}\left(\left|x_{i}-\widetilde{x}\right|\right) \\
S_{n}=1.1926 \operatorname{median}_{\mathrm{i}}\left(\operatorname{median}_{j}\left|x_{i}-x_{j}\right|\right) \\
Q_{n}=2.219\left\{\left|x_{i}-x_{j}\right| ; i<j\right\}_{(k)}
\end{array}\right.
$$

where $\mathrm{x}_{\mathrm{i}}=$ sample value; $\widetilde{\mathrm{x}}=$ sample median; $\mathrm{k}=\mathrm{h}(\mathrm{h}-1) / 2 ; \mathrm{h}=[\mathrm{n} / 2]+1 ;$ and $[\mathrm{]}=$ integer 
This parameter replacement is justified by the breakdown point which is the largest fraction of arbitrary gross errors tolerated before the statistic becomes totally unreliable (Hampel, 2001). The new location and scale estimators have a breakdown point of $50 \%$ against $0 \%$ of the mean and standard deviation used by the normal z-score test.

The method, using all scale estimators, was applied to generated random samples and the finest results were achieved using the $S_{n}$ parameter.

A value is marked as an outlier if $Z_{i}^{*}$ is greater than a critical value that depends on the dimension of the sample and of the confidence level. After identifying the outliers they are removed and the median of the remaining records of the sample is calculated.

However if a large number of outliers id detected in a sample, that sample should be ignored and the readings must be repeated.

\subsection{Data estimation}

Data can be estimated by applying measured input of the bridge, like traffic loads, temperature or wind to the analytic model and calculating the expected value of the variables monitored. Another form of predicting a measure is using the correlations between several measurements.

With forecasted values and uncertainty margins upper and lower limits are defined. These limits are used to verify validated data.

\section{SALGUEIRO MAIA BRIDGE}

\subsection{Description of the structure}

The Salgueiro Maia Bridge is a cable-stayed bridge and has a total length of $570 \mathrm{~m}$ divided by seven spans with a main span of $246 \mathrm{~m}$ (Figure 1).

The masts, with a height of $50 \mathrm{~m}$ above the deck, are in reinforced concrete up to the level of the first cables, and from there on have a steel-concrete composite structure. The two masts are monolithic with the deck.

The stay cables support the bridge deck and are anchored at the masts and at the deck axis. Each mast has 36 cables with lengths that vary from $31 \mathrm{~m}$ up to $131 \mathrm{~m}$. The number of tendons per cable is also variable and may be 55,61 or 73 .

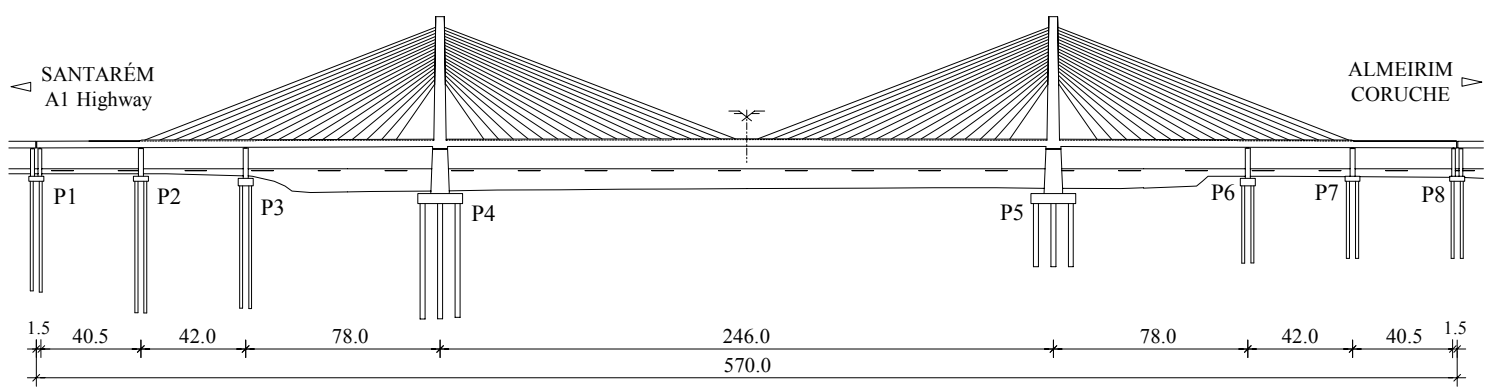

Figure 1. Longitudinal section of Salgueiro Maia cable-stayed bridge

The bridge deck has a cross-section formed by a $10.0 \mathrm{~m}$ by $2.5 \mathrm{~m}$ hollow rectangular shape caisson with an upper lateral slab and pre-cast concrete bracing's.

The transference of stresses at the box-girder webs was done with diagonal steel elements that were placed connecting the bottom corners of the caisson to the middle axis of the upper slab (Figure 2).

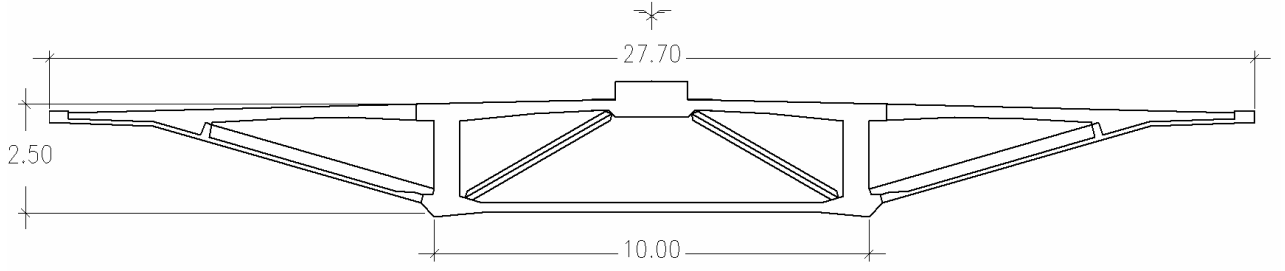

Figure 2. View of the cross-section of the bridge deck 


\subsection{Bridge monitoring system}

During and after bridge construction, several sections were instrumented (Figure 3a). Over 200 sensors (vibrating strain-wires and resistance thermometers) were installed inside the concrete at the pylons and at the bridge deck to monitor strains, temperature as well as the evolution of time dependent effects as creep or shrinkage; approximately 50 electrical strain gauges were installed at the deck steel ties.

Weather stations were mounted to measure air temperature and its relative humidity.

Beside the sensors mentioned above it were also installed air-bubble clinometers to evaluate rotations, at the top of the pylons and along the deck, and active measuring bases to control the evolution of joint movement (Figure 3b).

At the end of the construction, it was installed a hydrostatic levelling system associated to pressure cells to measure vertical displacements of the deck and force cells to measure cable tensions (Figure 3c).

A local network of 10 data-loggers was implemented to make the acquisition of the data. The transference of the data is made via modem (Figure 3d).

In situ data processing is made by the data-loggers, as for the main analysis, graphical presentation and report preparation is done in the office.

Before it was opened to the traffic, the bridge was subjected to static and dynamic load tests (Santos et al. 2004).

a) Instrumented sections

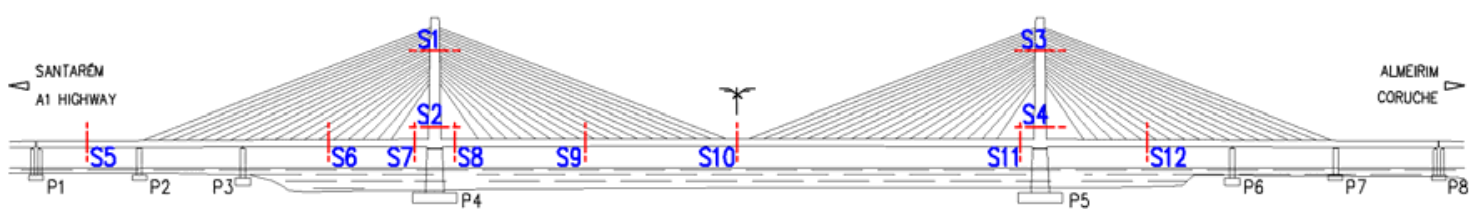

b) Air-bubble clinometers and joint meter bases

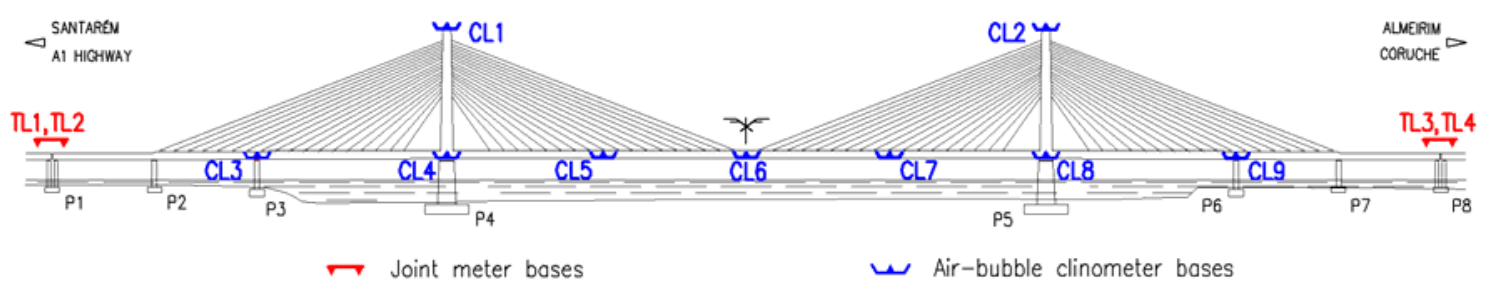

c) Load and pressure cells

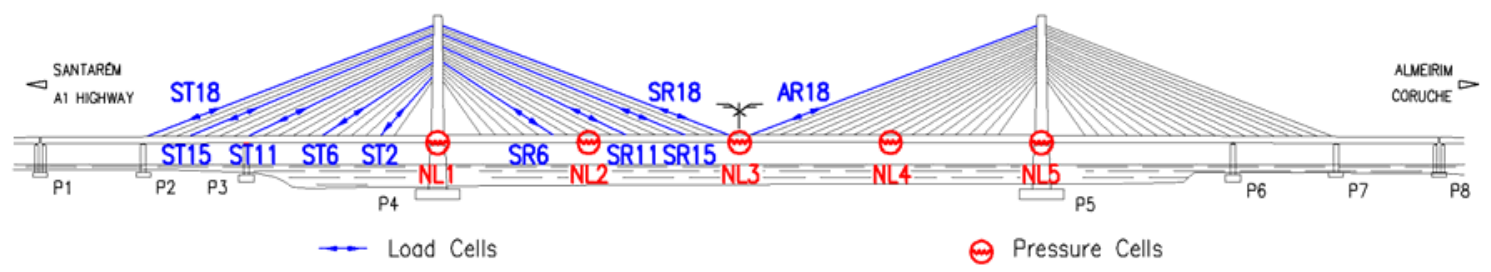

d) Data-logger network

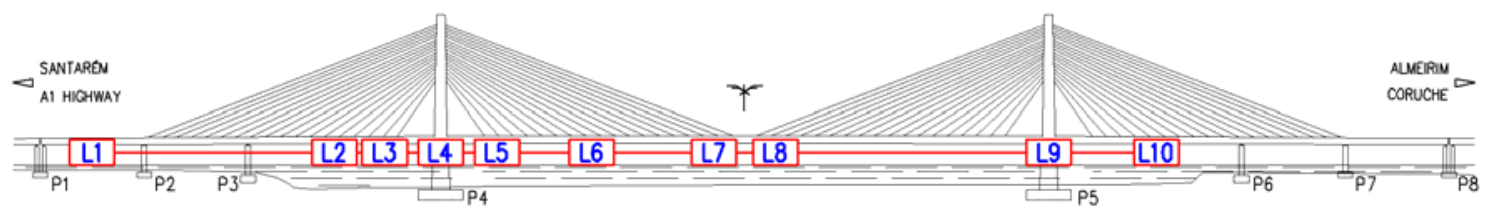

Figure 3. Location of Salgueiro Maia monitoring system equipment 


\subsection{Vertical displacements measurement}

A hydrostatic levelling system associated with pressure cells was implemented to measure vertical displacements of the main span of the bridge deck.

The pressure cells are located at piers P4 and P5, at mid-span and quarter-spans (Figure 3c), the deposit is situated at pier P4 and the signals of pressure cells are being acquired by a data-logger (Figure 4).

The relative vertical displacements are calculated with the variation of cells pressures using cells NL1 and NL5 as fixed references.
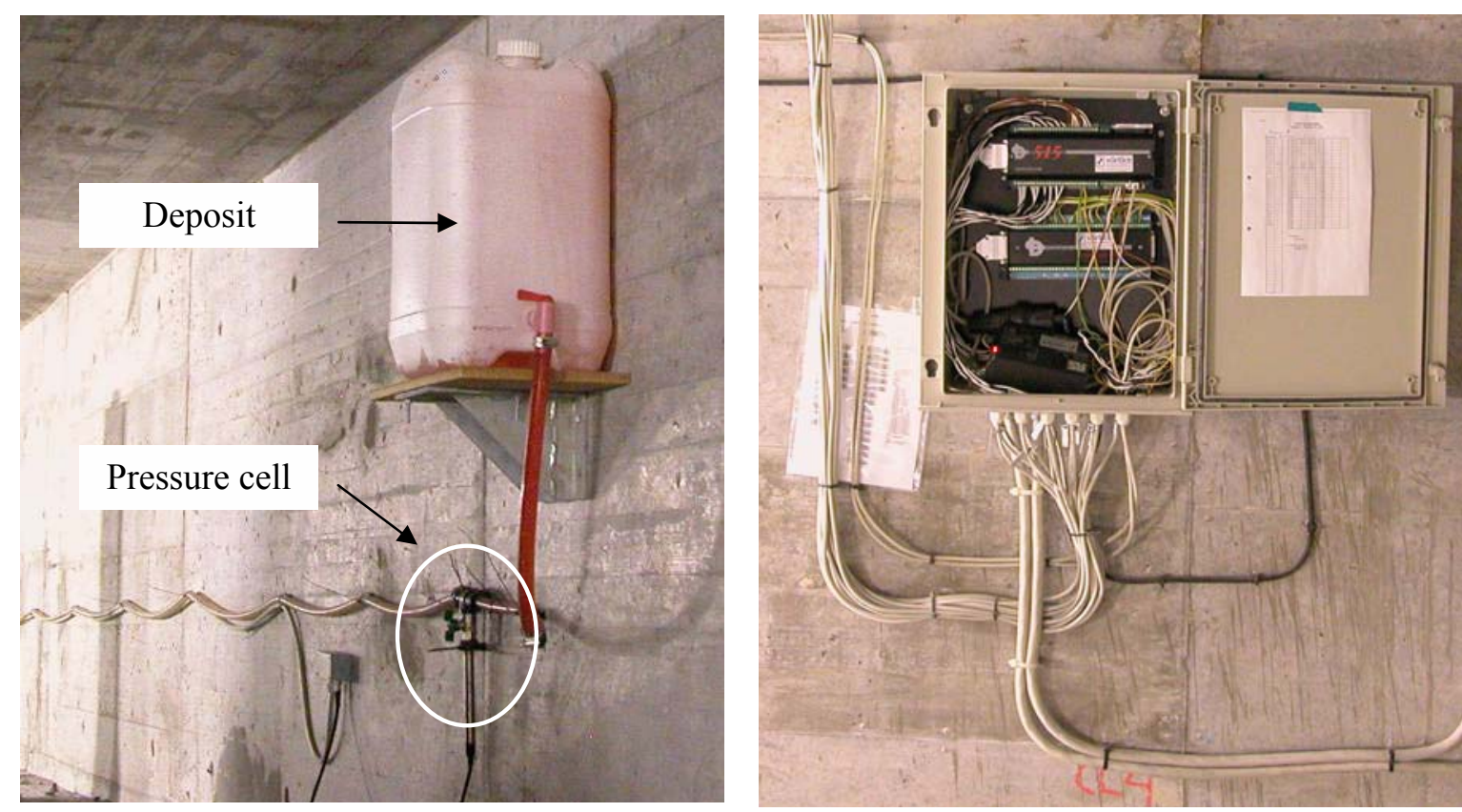

Figure 4. Hydrostatic levelling system (on the left pier P4, on the right data-logger acquisition system)

Due to the flexibility of cable stayed bridges, and also to the technique used to measure the displacements, the response of the structure has some small variation, for instance, due to traffic loads (Figure 5). The application of analysis procedure to these measurements would origin spurious alarms as a result of nuisance data. So, before data processing it is necessary to validate the measured values.

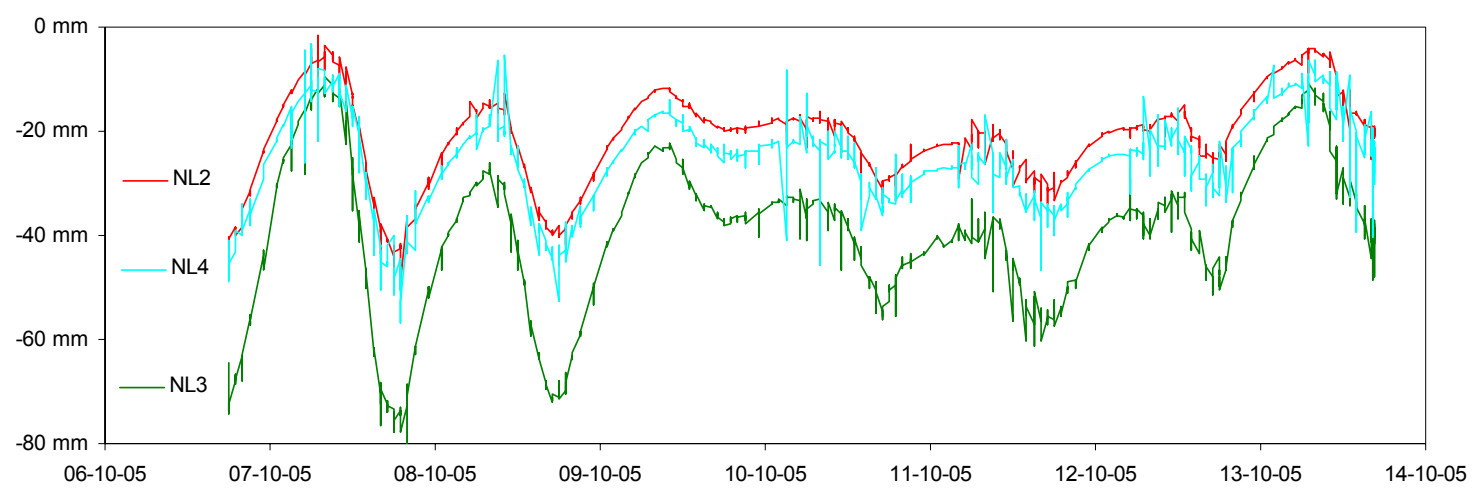

Figure 5 - Raw data of vertical displacements of main span 


\subsection{Vertical Displacements Validation}

The validation of the data was done using the method Z-Score modified with $\mathrm{S}_{\mathrm{n}}$, equation (2). Each sample had only 6 measurements so a multiplicative correction factor for finite populations was applied, in this case $c_{n}=0.992$.

$$
\mathrm{Z}_{\mathrm{i}}^{*}=\frac{\left|\mathrm{x}_{\mathrm{i}}-\widetilde{\mathrm{x}}\right|}{\mathrm{S}_{\mathrm{n}}} \quad \mathrm{S}_{\mathrm{n}}=\mathrm{c}_{\mathrm{n}} 1.1926 \operatorname{median}_{\mathrm{i}}\left(\operatorname{median}_{\mathrm{j}}\left|\mathrm{x}_{\mathrm{i}}-\mathrm{x}_{\mathrm{j}}\right|\right)
$$

where $\widetilde{\mathrm{x}}=$ sample median; and $\mathrm{c}_{\mathrm{n}}=$ correction factor for sample size

A value was marked as an outlier for $Z_{i}^{*}>1.944$ which is the critical value for $n=6$ and $99 \%$ confidence level (Burke, 2001). All the outliers were eliminated and afterwards the median of the remaining records of the sample was calculated. In Figure 6 it is possible to see the efficiency of the method.

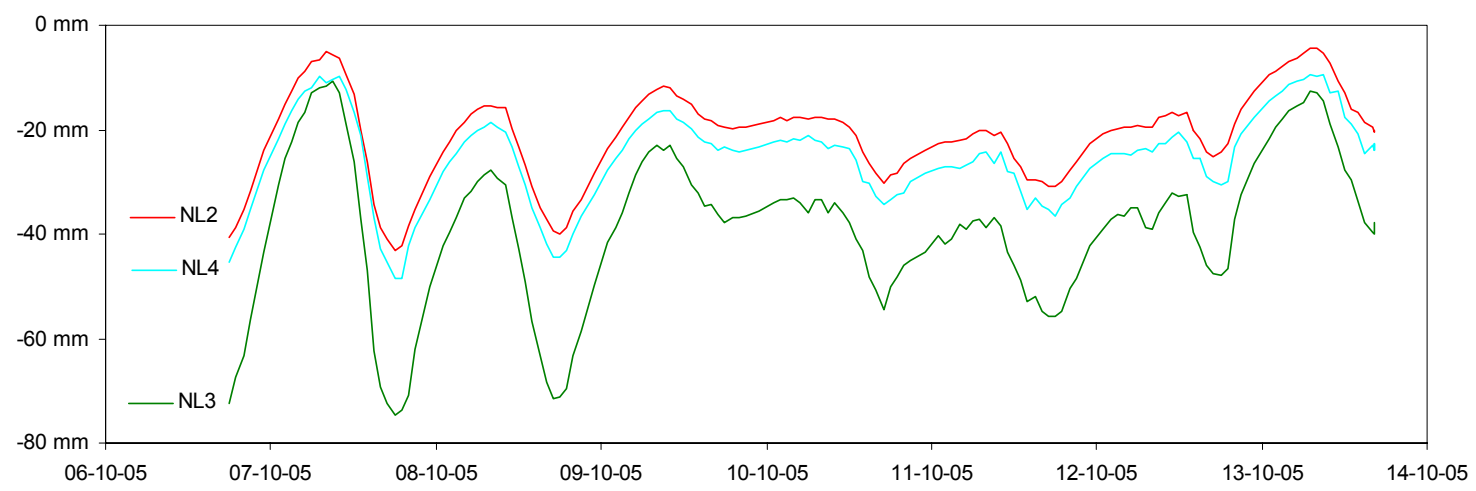

Figure 6 - Median of cleaned data of vertical displacements of main span

\subsection{Vertical Displacements Estimation}

The vertical displacements of a bridge depend of several factors, such as traffic loads, temperature, rotation of piers and masts, cable forces, creep or shrinkage. In the current section it is presented the estimation of vertical displacements based on its correlation with temperature.

On Figure 7 is represented the air temperature measured on the mid-span on the period in analysis. By observing this figure it is noticeable that whenever the temperature increases the displacement also increases and vice-versa.

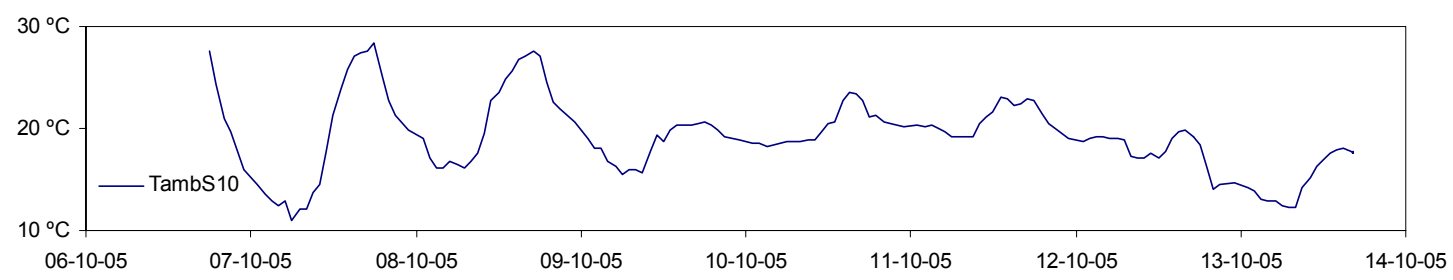

Figure 7 - Air Temperature in S10

However, in a closer look of both graphics (Figure 6 and Figure 7), it is detected that the picks of air temperature don't mach the displacement picks. This observation corresponds to what is predictable due to the slow reaction of concrete structures to temperature effects. The following graphics show the correlations between air temperature and vertical displacements using different gaps between related values. 
Air Temperature Vs. Displacement (after 1 Hour)
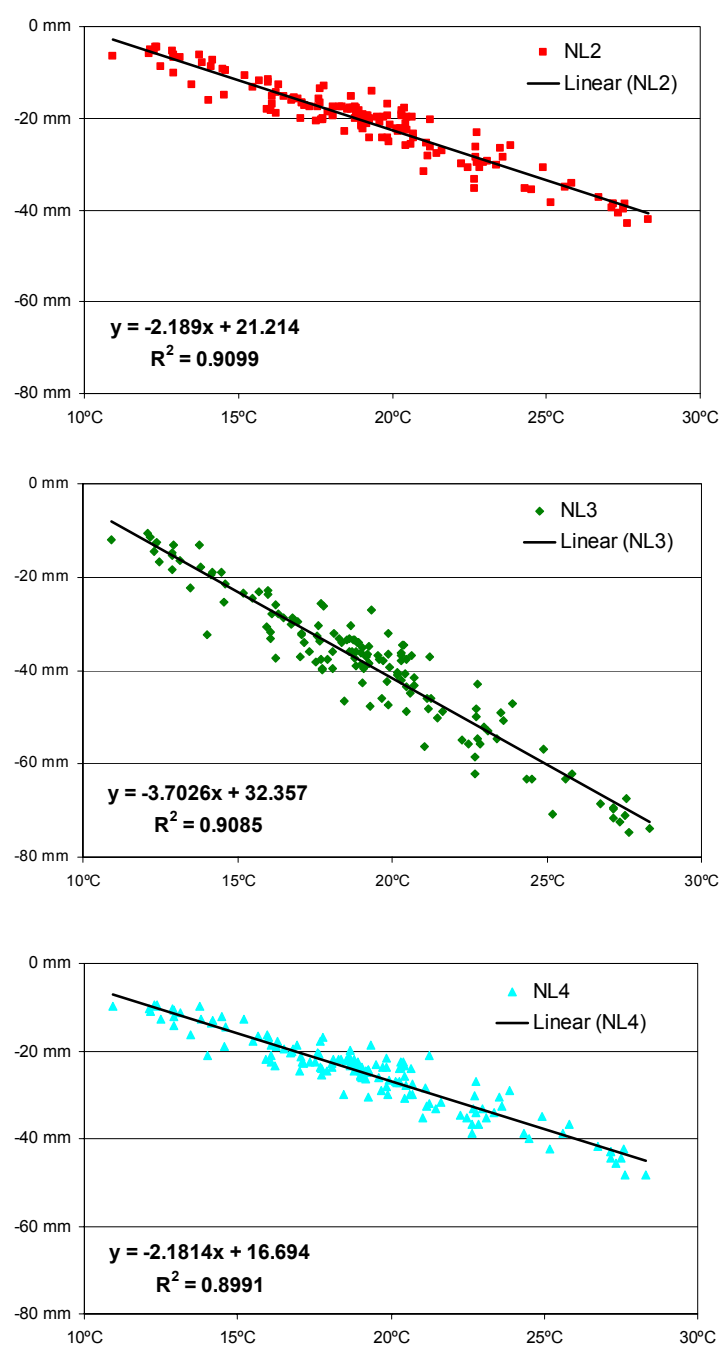

Air Temperature Vs. Displacement (after 2 Hour)
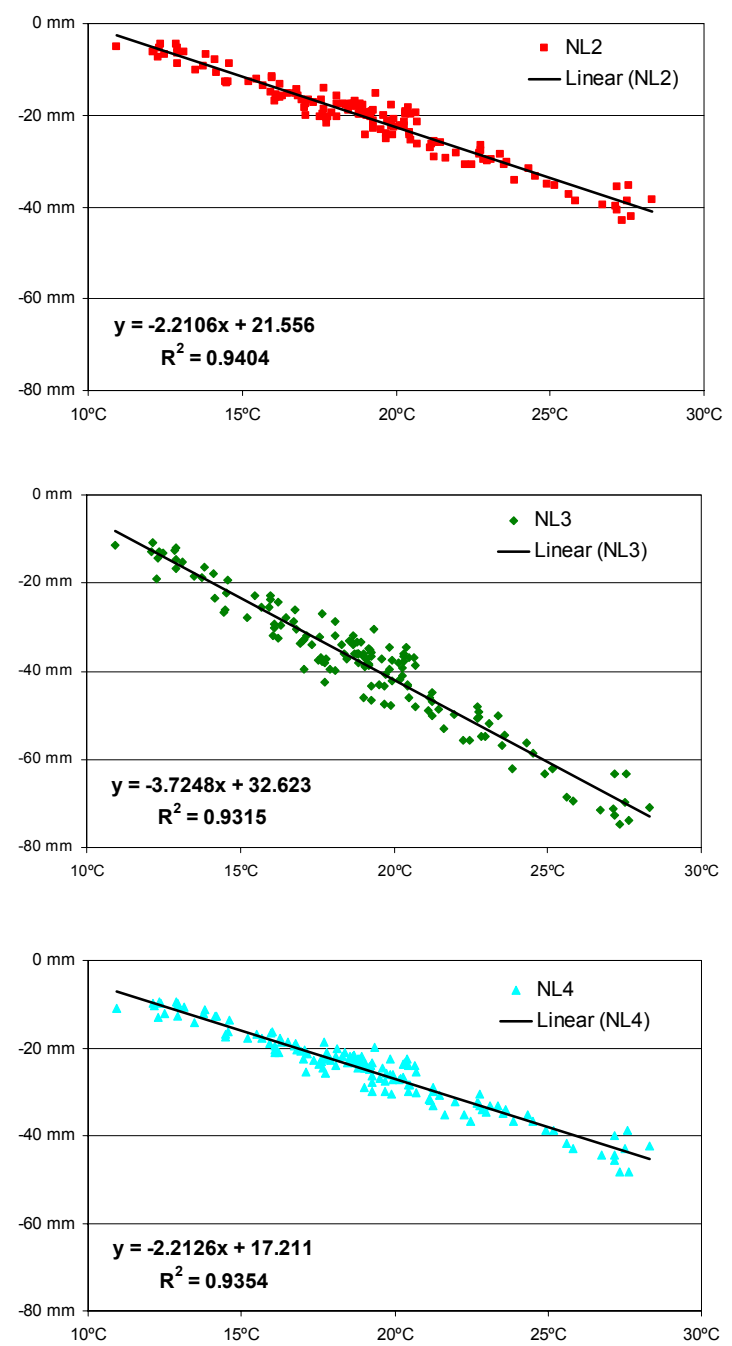

Figure 8 - Correlation of air temperature and vertical displacements (on left 1 hour gap, on right 2 hours gap)

The estimated values were calculated using the equations determined from the correlation with the 2 hours gap (Figure 9), which presented an average correlation factor of 0.93 .

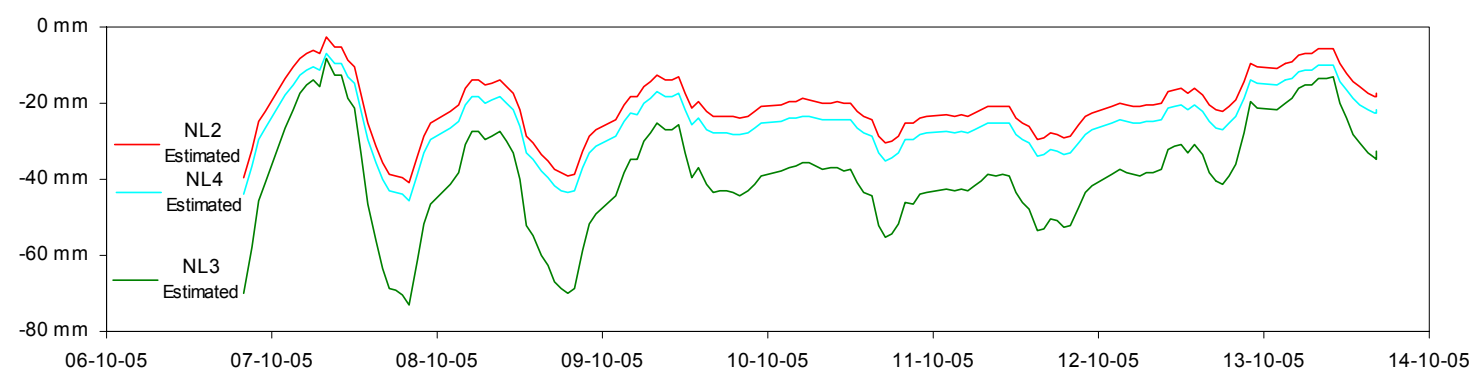

Figure 9 - Estimated vertical displacements

On the following graphics the measured vertical displacements are verified using the estimated values to determine minimum and maximum limits using an uncertainty margin of $10 \mathrm{~mm}$. 

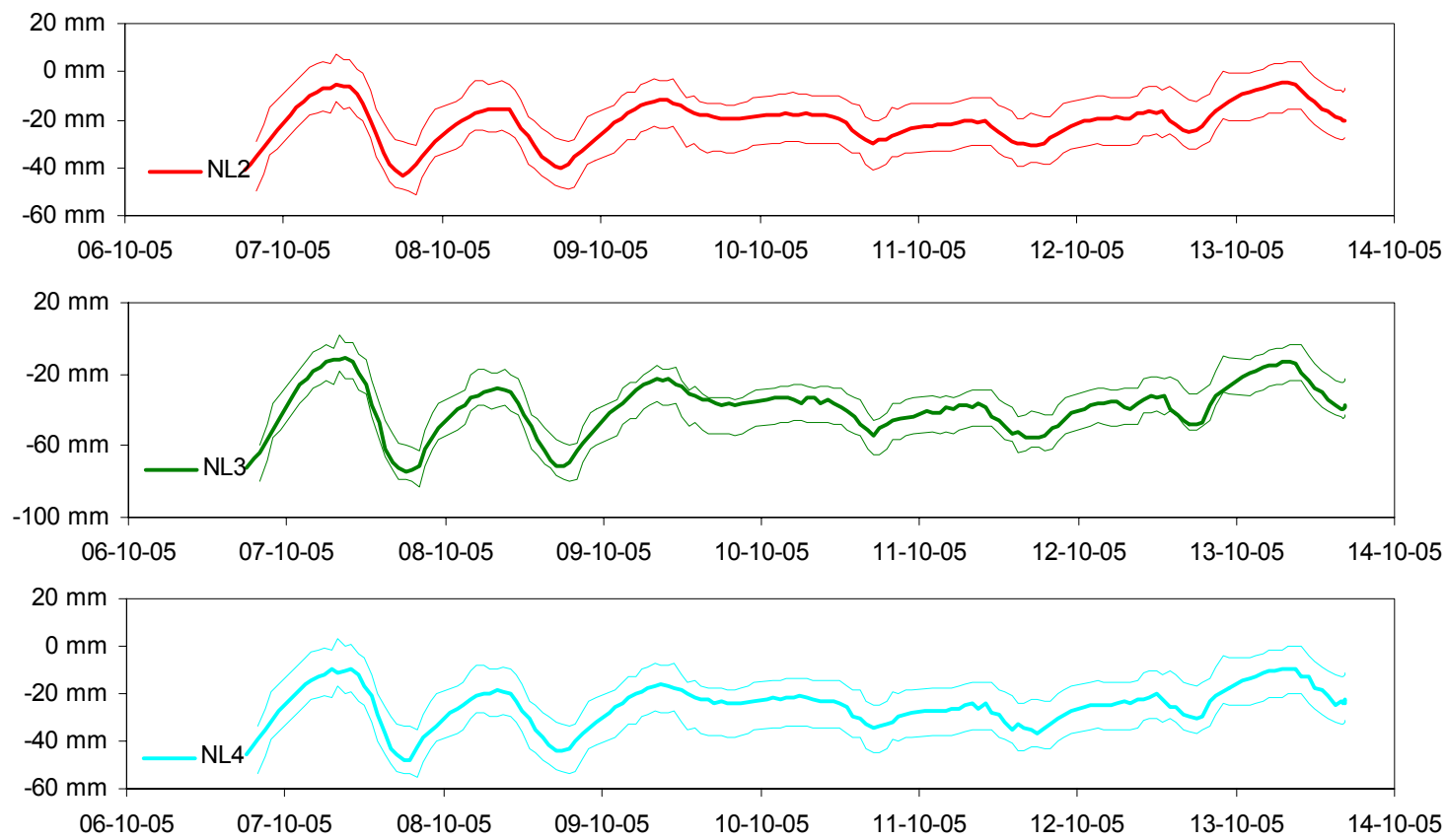

Figure 10 - Measured displacements within estimated ranges using uncertainty margins of $10 \mathrm{~mm}$

\section{CONCLUSIONS}

New developments in sensors and the upgrading of storage and acquisition equipment lead to the necessity of new methodologies for data processing.

For data management and analysis procedures it is needed to have reliable measurements from each sensor and also from the relations between different sensors.

This paper shows the efficiency of modified z-score method, with the use of $S_{n}$ to estimate scale, on the validation of vertical displacement.

With the use of measured air temperature it was possible to forecast the displacements in order to create ranges for verification of measured values.

For future analysis the use of combine correlations between other monitored data and also the use of a finite element model would result on better predictions for measured data that could reduce uncertainty margins.

\section{REFERENCES}

Aktan, A.E. \& Catbas, F.N. \& Grimmelsman, K. \& Pervizpour, M. \& Curtis, J. \& Shen, K. \& Qin, X. 2002. Sensing, Communication, Computing And Information Systems For Infrastructure Health, Performance And Security Monitoring. http://www.di3.drexel.edu/

Burke, S. 2001. Missing Values, Outliers, Robust Statistics \& Non-parametic Methods. Statistics and data analysis. $L G-C G$ Europe online supplement N. 59.

Datataker 2005. Powerful Data Logger and Flexible Data Acquisition \& Data Logging Systems. http://www.datataker.com/acquisition.html

Marecos, V.M. \& Santos, L.O. \& Branco, F.A. Bridges safety control in real time 2004. Static and dynamic tests of Salgueiro Maia cable-stayed bridge. Proceedings of the Second international Conference on Bridge Maintenance, Safety and Management, IABMAS'04, Kyoto.

Santos, L.O. \& Rodrigues, J. \& Min, X. \& Fernandes, J. A. 2004. Static and dynamic tests of Salgueiro Maia cable-stayed bridge. Proceedings of the Second international Conference on Bridge Maintenance, Safety and Management, IABMAS'04, Kyoto.

Zhang, Y. \& Luo, A. \& Zhao, Y. 2004. Outlier detection in astronomical data. Optimizing scientific return for astronomy through information technologies, Glasgow, Scotland United Kingdom: SPIE. 